\title{
Minimum Cross Fuzzy Entropy Problem, The Existence of Its Solution and Generalized Minimum Cross Fuzzy Entropy Problems
}

\author{
Aladdin Shamilov, Nihal İnce* \\ Faculty of Science, Department of Statistics, Anadolu University, 26470, Eskisehir, Turkey.
}

Received: June 13, 2016 / Accepted: July 10, 2016 / Published: August 25, 2016.

\begin{abstract}
In the present study we have formulated a Minimum Cross Fuzzy Entropy Problem (Minx(F)EntP) and proposed sufficient conditions for existence of its solution. Mentioned problem can be formulated as follows. In the set of membership functions satisfying the given moment constraints generated by given moment functions it is required to choose the membership function that is closest to a priori membership function in the sense of cross fuzzy entropy measure. The existence of solution of formulated problem is proved by virtue of concavity property of cross fuzzy entropy measure, the implicit function theorem and Lagrange multipliers method. Moreover, Generalized Cross Fuzzy Entropy Optimization Methods in the form of MinMinx(F)EntM and MaxMinx(F)EntM are suggested on the basis of primary phase of minimizing cross fuzzy entropy measure for fixed moment vector function and on the definition of the special functional with Minx(F)Ent values of cross fuzzy entropy measure. Next phase for obtaining mentioned distributions consists of optimization of defined functional with respect to moment vector functions. Distributions obtained by mentioned methods are defined as $(\operatorname{MinMinx}(\mathrm{F}) \text { Ent })_{\mathrm{m}}$ and $(\operatorname{MaxMinx}(\mathrm{F}) \text { Ent })_{\mathrm{m}}$ distributions.
\end{abstract}

Keywords: Cross fuzzy entropy measure, Generalized fuzzy entropy optimization problem, Existence theorem

\section{Introduction}

The concept of entropy plays a significant role in measurement of the degree of uncertainity and many problems concerned with its applications are given in [4]. First, Zadeh [2,3] introduced the fuzzy entropy using the concept of membership function. Then, De Luca and Termini [7] defined entropy measure of a fuzzy set based on Shannon's function [6]. After these developments, a large number of measures of fuzzy entropy were discussed, characterized and generalized by various authors. Some other interesting findings related with theoretical measures of fuzzy entropy and their applications have been provided by Kapur [5], Parkash and Sharma [13], Yager [14], Bhandari and Pal [8] etc. The starting point for the cross entropy

Corresponding author: Nihal İnce, Faculty of Science, Department of Statistics, Anadolu University, 26470, Eskisehir, Turkey. E-mail: nihalyilmaz@anadolu.edu.tr. approach is information theory developed by Shannon [6]. Kullback-Leibler [16] suggested the principle of minimum cross entropy measure. Optimization problems of Jaynes and Kullback-Leibler measures subject to constraints generated by given moment functions have many applications in different scientific fields as information theory, statistics and etc.

Corresponding to De Luca and Termini's [7] fuzzy entropy measure, Bhandari and Pal [8] introduced the cross entropy for a fuzzy set using its membership function. In addition, some new information measures and cross-entropy of fuzzy sets have been proposed in [9-12].

In our study we have formulated a new Minimum Cross Fuzzy Entropy Problem (Minx(F)EntP) and proposed sufficient conditions for existence of its solution. Moreover, according to [17-21] we have suggested new Generalized Cross Fuzzy Entropy 
Optimization Methods in the form of MinMinx(F)EntM and MaxMinx(F)EntM which are different from that of [9-13].

The suggested research is formed as follows. In Section 2, the statement of Fuzzy Entropy Optimization Problem and its solving method are proposed. In Section 3, the concavity of cross fuzzy entropy measure is proved. In Section 4, the Existence theorem for solution of Cross Fuzzy Entropy Optimization Problem is proved. In Section 5, the evaluation of Lagrange multipliers for Minimum Cross Fuzzy Entropy Problem is formulated. In Section 6 , the minimum cross fuzzy entropy value via Lagrange multipliers is achieved. In Section 7, new Generalized Cross Fuzzy Entropy Optimization problems $(\operatorname{MinMinx}(\mathrm{F}) \mathrm{Ent})_{\mathrm{m}},(\operatorname{Max} \operatorname{Minx}(\mathrm{F}) \mathrm{Ent})_{\mathrm{m}}$ and methods of solving these problems are developed. Finally, the main results obtained in this study are summarized.

\section{Minimum Cross Fuzzy Entropy Problem (Minx(F)EntP)}

According to definition of membership function of fuzzy set $F$ denoted by $\mu_{F}$ in crisp set maps whole members in universal set $X$ to $\{0,1\}, \mu_{F}: X \rightarrow\{0,1\}$. However, in fuzzy sets, each element is mapped to $[0,1]$ by membership function, $\mu_{F}: X \rightarrow[0,1]$. For this reason, fuzzy set can be described as "vague boundary" set comparing with crisp set in [22].

Let $A, B$ be fuzzy sets with membership functions $\mu_{A}\left(x_{i}\right), \mu_{B}\left(x_{i}\right), i=0,1, \ldots n$, respectively. Then, corresponding to Kullback-Leibler probabilistic entropy [16] the measure $D\left(\mu_{A}: \mu_{B}\right)$ of cross fuzzy entropy for fuzzy sets [10] $A, B$ containing finite number elements can be expressed by formula

$$
\begin{aligned}
D\left(\mu_{A}: \mu_{B}\right)= & {\left[\sum_{i=0}^{n} \mu_{A}\left(x_{i}\right) \ln \frac{\mu_{A}\left(x_{i}\right)}{\mu_{B}\left(x_{i}\right)}+(1\right.} \\
& \left.\left.-\mu_{A}\left(x_{i}\right)\right) \ln \left(\frac{1-\mu_{A}\left(x_{i}\right)}{1-\mu_{B}\left(x_{i}\right)}\right)\right],
\end{aligned}
$$

where

$$
\begin{aligned}
& \mu_{A}=\left(\mu_{A}\left(x_{0}\right), \mu_{A}\left(x_{1}\right), \ldots, \mu_{A}\left(x_{n}\right)\right. \\
& \mu_{B}=\left(\mu_{B}\left(x_{0}\right), \mu_{B}\left(x_{1}\right), \ldots, \mu_{B}\left(x_{n}\right)\right.
\end{aligned}
$$

$\operatorname{Minx}(\mathrm{F})$ EntP consists of minimizing cross fuzzy entropy measure (1) with respect to membership functions $\mu_{A}(x)$ with finite number of the fuzzy values $\mu_{A}\left(x_{i}\right), i=0,1, \ldots, n$ and a priori membership function $\mu_{B}(x)$ with finite number of the fuzzy values $\mu_{B}\left(x_{i}\right), i=0,1, \ldots, n$ subject to constraints,

$$
\sum_{i=0}^{n} \mu_{A}\left(x_{i}\right) g_{j}\left(x_{i}\right)=\mu_{j}, j=0,1,2, \ldots, m
$$

where $g_{0}(x) \equiv 1 ; \mu_{j}, j=0,1,2, \ldots, m$ are moment values of $\mu_{A}\left(x_{i}\right), i=0,1, \ldots, n$ with respect to moment functions $g_{j}(x), j=0,1,2, \ldots, m ; m<n$.

Mentioned problem can be formulated as follows. In the set of membership functions satisfying the given moment constraints generated by given moment functions it is required to choose the membership function that is closest to a priori membership function in the sense of minimum cross fuzzy entropy measure.

The distribution of fuzzy values $\left(\mu_{A}\left(x_{0}\right), \mu_{A}\left(x_{1}\right), \ldots, \mu_{A}\left(x_{n}\right)\right)$ minimizing function (1) subject to constraints (2) (briefly stated problem (1),(2)) we call Minimum Cross Fuzzy Entropy Distribution (Minx(F)EntD) just as Minimum Cross Entropy Distribution (MinxEntD) of probabilistic entropy optimization problem [19].

Minimum Cross Fuzzy Entropy Problem (1),(2) is a conditional extremum problem. Solvability of this problem is required to fulfillment of several conditions. Mentioned conditions are following:

(1) Moment functions $g_{j}(x), j=0,1,2, \ldots, m$ are linearly independent;

(2) The inequality $n>m$ is satisfied;

(3) Moment values $\tilde{\mu}_{j}, j=0,1,2, \ldots, m$ are obtained by virtue of given fuzzy values $\tilde{\mu}_{A}\left(x_{i}\right), i=$ $0,1, \ldots, n$ and moment functions $g_{j}(x), j=0,1, \ldots, m$ in the form of equalities 


$$
\sum_{i=0}^{n} g_{j}\left(x_{i}\right) \tilde{\mu}_{A}\left(x_{i}\right)=\tilde{\mu}_{j}, j=0,1, \ldots, m
$$

Remark. (2 $\left.2_{1}\right)$ means that there are linear dependency between the column $\tilde{\mu}=\left(\tilde{\mu}_{0}, \tilde{\mu}_{1}, \ldots, \tilde{\mu}_{m}\right)^{T}$ and all columns of matrix $A=\left(g_{j}\left(x_{i}\right)\right)_{\substack{j=0,1, \ldots, m \\ i=0,1, \ldots, n}}$ Consequently, $\operatorname{rank} A$ is equal to $\operatorname{rank}(A: \tilde{\mu})$, where $(A: \tilde{\mu})$ is augmented matrix for system (2) as the matrix $A$ with column $\tilde{\mu}$ added to it. Therefore, system (2) with respect to $\mu_{A}\left(x_{i}\right), i=0,1, \ldots, n$ has a solution. Note that from condition 1) follows that $\operatorname{rank} A=m+1$.

\section{Concavity of Cross Fuzzy Entropy}

\section{$\operatorname{Measure}\left(D\left(\mu_{A}: \mu_{B}\right)\right)$}

In order to simplify mathematical operations constrained with (1) we use conventional signs $X_{i}=\mu_{A}\left(x_{i}\right) ; A_{i}=\mu_{B}\left(x_{i}\right), i=0,1, \ldots, n$ and write (1) in the form

$$
D(X: A)=\sum_{i=0}^{n}\left[X_{i} \ln \frac{X_{i}}{A_{i}}+\left(1-X_{i}\right) \ln \left(\frac{1-X_{i}}{1-A_{i}}\right)\right],
$$

where $X=\left(X_{0}, X_{1}, \ldots, X_{n}\right)$ ve $A=\left(A_{0}, A_{1}, \ldots, A_{n}\right)$.

From (1') follows that

$$
\begin{gathered}
\frac{\partial D}{\partial X_{i}}=\ln \left(\frac{X_{i}}{1-X_{i}} \frac{1-A_{i}}{A_{i}}\right) \\
\frac{\partial^{2} D}{\partial X_{i} \partial X_{j}}=\left\{\begin{array}{c}
\frac{1}{X_{j}\left(1-X_{j}\right)}, i=j . \\
0, i \neq j
\end{array}\right.
\end{gathered}
$$

Consequently, Hessian matrix $\mathcal{H}$ is defined in the form

$$
\mathcal{H}=\left(\frac{\partial^{2} D}{\partial X_{i} \partial X_{j}}\right)_{\substack{j=0,1 \ldots, n \\ i=0,1, \ldots, n}}
$$

Since eigenvalues of $\mathcal{H}$ matrix are positive: $\frac{1}{X_{i}\left(1-X_{i}\right)}>0, i=0,1, \ldots, n$, therefore this matrix is positive defined. This result shows that function $D$ is concav and at critical point $\left(X_{0}^{0}, X_{1}^{0}, \ldots, X_{n}^{0}\right)$,
$X_{i}^{0}=A_{i}$ for which $\frac{\partial D}{\partial X_{i}}=0$ function $D$ reaches minimum.

\section{The existence of solution of Minimum Cross Fuzzy Entropy Problem (Minx(F)EntP)}

$\operatorname{Minx}(\mathrm{F})$ EntP (1),(2) is a conditional extremum problem and can be solved by Lagrange multipliers method. According to Lagrange multipliers method firstly the new auxiliary function $U$ is constructed:

$$
\begin{aligned}
U=\sum_{i=0}^{n}\left[\mu_{A}\left(x_{i}\right)\right. & \ln \frac{\mu_{A}\left(x_{i}\right)}{\mu_{B}\left(x_{i}\right)}+(1 \\
& \left.-\mu_{A}\left(x_{i}\right)\right) \ln \left(\frac{1-\mu_{A}\left(x_{i}\right)}{1-\mu_{B}\left(x_{i}\right)}\right) \\
& -\sum_{j=0}^{m} \lambda_{j}\left(\sum_{i=1}^{n} \mu_{A}\left(x_{i}\right) g_{j}\left(x_{i}\right)\right. \\
& \left.-\mu_{j}\right)
\end{aligned}
$$

where $\lambda_{j}$ are certain constant factors and the function $U$ is now investigated for an unconditional extremum; we form a system of equations $\frac{\partial U}{\partial \lambda_{j}}=0, j=$ $0,1, \ldots, m$ supplemented by the constraint equations (2) from which all the $n+m+2$ unknowns $\mu_{A}\left(x_{i}\right), i=0,1, \ldots, n \quad$ and $\quad \lambda_{j}, j=0,1, \ldots, m \quad$ are determined.

From (3) follows that

$$
\begin{gathered}
\frac{\partial U}{\partial \mu_{A}\left(x_{i}\right)}=\ln \frac{\mu_{A}\left(x_{i}\right)}{\mu_{B}\left(x_{i}\right)}+\mu_{A}\left(x_{i}\right) \frac{1}{\frac{\mu_{A}\left(x_{i}\right)}{\mu_{B}\left(x_{i}\right)}} \frac{1}{\mu_{B}\left(x_{i}\right)} \\
-\ln \left(\frac{1-\mu_{A}\left(x_{i}\right)}{1-\mu_{B}\left(x_{i}\right)}\right)+ \\
+\left(1-\mu_{A}\left(x_{i}\right)\right) \frac{1}{\frac{1-\mu_{A}\left(x_{i}\right)}{1-\mu_{B}\left(x_{i}\right)}} \frac{1}{1-\mu_{B}\left(x_{i}\right)}(-1) \\
-\sum_{j=0}^{m} \lambda_{j} g_{j}\left(x_{i}\right)=0
\end{gathered}
$$




$$
\begin{gathered}
\ln \frac{\mu_{A}\left(x_{i}\right)}{\mu_{B}\left(x_{i}\right)}+1-\ln \left(\frac{1-\mu_{A}\left(x_{i}\right)}{1-\mu_{B}\left(x_{i}\right)}\right)-1-\sum_{j=0}^{m} \lambda_{j} g_{j}\left(x_{i}\right) \\
=0 \\
\ln \frac{\mu_{A}\left(x_{i}\right)}{\mu_{B}\left(x_{i}\right)}-\ln \left(\frac{1-\mu_{A}\left(x_{i}\right)}{1-\mu_{B}\left(x_{i}\right)}\right)-\sum_{j=0}^{m} \lambda_{j} g_{j}\left(x_{i}\right)=0 \\
\mu_{A}\left(x_{i}\right)=\frac{1}{1+\frac{1-\mu_{B}\left(x_{i}\right)}{\mu_{B}\left(x_{i}\right)} e^{\sum_{j=0}^{m} \lambda_{j} g_{j}\left(x_{i}\right)}} \\
i=0,1, \ldots, n .
\end{gathered}
$$

Also, from (1) follows that

$\frac{\partial U}{\partial \lambda_{j}}=-\left(\sum_{j=0}^{m} \mu_{A}\left(x_{i}\right) g_{j}(x)-\mu_{j}\right)=0, j=0,1, \ldots, m$

It is seen that the equalites (4) are constraint equations (2). If we take (4) into account in (2), then

$$
\begin{gathered}
\sum_{i=0}^{n} \frac{1}{1+\frac{1-\mu_{B}\left(x_{i}\right)}{\mu_{B}\left(x_{i}\right)} e^{\sum_{j=0}^{m} \lambda_{j} g_{j}\left(x_{i}\right)}} g_{j}\left(x_{i}\right)=\mu_{j}, j \\
=0,1,2, \ldots, m
\end{gathered}
$$

If denote the left-hand of (5) by $f_{k}\left(\lambda_{0}, \lambda_{1}, \ldots, \lambda_{m}\right)$, then (5) can be written as

$$
\begin{aligned}
& f_{k}\left(\lambda_{0}, \lambda_{1}, \ldots, \lambda_{m}\right) \\
& =\sum_{i=0}^{n} \frac{1}{1+\frac{1-\mu_{B}\left(x_{i}\right)}{\mu_{B}\left(x_{i}\right)} e^{\sum_{j=0}^{m} \lambda_{j} g_{j}\left(x_{i}\right)}} g_{k}\left(x_{i}\right)=\mu_{k}, k \\
& =0,1, \ldots, m
\end{aligned}
$$

From $\left(2_{1}\right)$ follows that

$$
\begin{aligned}
\sum_{i=0}^{m} g_{j}\left(x_{i}\right) \tilde{\mu}_{A}\left(x_{i}\right) & =\tilde{\mu}_{j}-\sum_{i=m+1}^{n} g_{j}\left(x_{i}\right) \tilde{\mu}_{A}\left(x_{i}\right), j \\
& =0,1, \ldots, m
\end{aligned}
$$

(7) shows that there are linear correlations between $\tilde{\mu}_{A}\left(x_{i}\right), \quad i=0,1, \ldots, m$ and $\tilde{\mu}_{A}\left(x_{i}\right), i=m+1, \ldots, n$. Consequently, from the assumption 1), that moment functions $g_{j}(x), j=0,1, \ldots, m$ are linearly indepedent, so the satisfiability of condition

$$
\operatorname{det}\left(g_{j}\left(x_{i}\right)\right)_{\substack{j=0,1, . ., m \\ i=0,1, \ldots, m}} \neq 0
$$

follows.
In (7), $\tilde{\mu}_{A}\left(x_{i}\right), i=0,1, \ldots, m$ by Cramer method of solving linear nonhomojenus algebrical equations can be expressed via $\tilde{\mu}_{j}, j=0,1, \ldots, m$ and $\tilde{\mu}_{A}\left(x_{i}\right), i=$ $m+1, \ldots, n$ in the form

$$
\begin{aligned}
& \tilde{\mu}_{A}\left(x_{i}\right) \\
& =F\left(\tilde{\mu}_{0}, \tilde{\mu}_{1}, \ldots, \tilde{\mu}_{m}, \tilde{\mu}_{A}\left(x_{m+1}\right), \ldots, \tilde{\mu}_{A}\left(x_{n}\right)\right), i \\
& =0,1, \ldots, m
\end{aligned}
$$

From (4) follows that

$$
\begin{aligned}
\sum_{j=0}^{m} \lambda_{j} g_{j}\left(x_{i}\right)= & \ln \left(\frac{1-\mu_{A}\left(x_{i}\right)}{\mu_{A}\left(x_{i}\right)} \frac{\mu_{B}\left(x_{i}\right)}{1-\mu_{B}\left(x_{i}\right)}\right), i \\
& =0,1, \ldots, m
\end{aligned}
$$

If subsitute $\left(2_{3}\right)$ in (8) and solve the getting equations with respect to $\lambda_{0}, \lambda_{1}, \ldots, \lambda_{m}$, then it is possible to find $\tilde{\lambda}_{0}, \tilde{\lambda}_{1}, \ldots, \tilde{\lambda}_{m}$ satisyfing (6). Then, the equations

$$
f_{k}\left(\tilde{\lambda}_{0}, \tilde{\lambda}_{1}, \ldots, \tilde{\lambda}_{m}\right)=\tilde{\mu}_{k}, \quad k=0,1, \ldots, m .
$$

are arised.

Therefore, subject to assumption $\left(2_{2}\right)$ by solving (7) with respect to $\tilde{\mu}_{A}\left(x_{i}\right), i=0,1, \ldots, m$ and substituting $\left(2_{3}\right)$ in (8) the relations (9) is appeared.

Note that relations (9) are one of the important conditions to solve equations (6) with repect to $\lambda_{0}, \lambda_{1}, \ldots, \lambda_{m}$. The other condition to solve equations (6) with respect to $\lambda_{0}, \lambda_{1}, \ldots, \lambda_{m}$ in the some neighbourhood of $\left(\tilde{\lambda}_{0}, \tilde{\lambda}_{1}, \ldots, \tilde{\lambda}_{m}\right)$ satisfiying (9) is the condition

$$
\frac{D\left(f_{0}, f_{1}, \ldots, f_{m}\right)}{D\left(\lambda_{0}, \lambda_{1}, \ldots, \lambda_{m}\right)} \neq 0
$$

Now, we prove the fulfillment of (10). From (6), we get

$$
\begin{gathered}
\frac{\partial f_{k}}{\partial \lambda_{l}}=\sum_{i=1}^{n} \mu_{A}\left(x_{i}\right)\left(1-\mu_{A}\left(x_{i}\right)\right) g_{k}\left(x_{i}\right) g_{l}\left(x_{i}\right) \\
k=0,1, \ldots, m, l=0,1, \ldots, m
\end{gathered}
$$

Let us $\sum_{i=0}^{n} \mu_{A}\left(x_{i}\right)\left(1-\mu_{A}\left(x_{i}\right)\right)=\alpha$. Then,

$$
\sum_{i=0}^{n} \frac{\mu_{A}\left(x_{i}\right)\left(1-\mu_{A}\left(x_{i}\right)\right)}{\alpha}=1
$$

In (12), we consider the ratio

$$
\frac{\mu_{A}\left(x_{i}\right)\left(1-\mu_{A}\left(x_{i}\right)\right)}{\alpha}
$$


as probability measure then

$$
\frac{\mu_{A}\left(x_{i}\right)\left(1-\mu_{A}\left(x_{i}\right)\right)}{\alpha}=P_{i}, i=0,1, \ldots, n ; \sum_{i=0}^{n} P_{i}=1
$$

and from (11) follows that

$$
\begin{gathered}
\frac{\partial f_{k}}{\partial \lambda_{l}}=\alpha \sum_{i=0}^{n} \frac{\mu_{A}\left(x_{i}\right)\left(1-\mu_{A}\left(x_{i}\right)\right)}{\alpha} g_{k}\left(x_{i}\right) g_{l}\left(x_{i}\right) \\
=\alpha \sum_{i=0}^{n} P_{i} g_{k}\left(x_{i}\right) g_{l}\left(x_{i}\right) \\
=\alpha E\left[g_{k} g_{l}\right]
\end{gathered}
$$

and

$$
\begin{aligned}
& R=\left(\frac{\partial f_{k}}{\partial \lambda_{l}}\right)_{k, l=0,1, \ldots, m}
\end{aligned}
$$

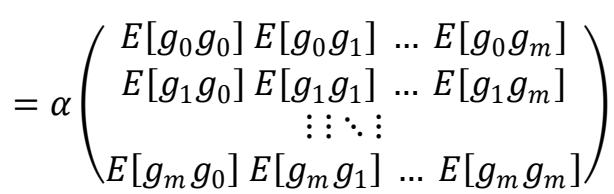

Since $R$ is correlation matrix of random variables $g_{0}(x), \ldots, g_{m}(x)$ each of which has $n+1$ number of values, then

$$
J=\frac{D\left(f_{0}, f_{1}, \ldots, f_{m}\right)}{D\left(\lambda_{0}, \lambda_{1}, \ldots, \lambda_{m}\right)}=\operatorname{det}(R) \neq 0
$$

Note that the satisfiability of last condition can be also proved as following:

$$
\begin{aligned}
0 \leq E\left\{\left|a_{0} g_{0}+a_{1} g_{1}+\cdots+a_{m} g_{m}\right|^{2}\right\} \\
=E\left\{\sum_{j=0}^{m} \sum_{k=0}^{m} a_{j} a_{k} g_{j} g_{k}\right\}= \\
=\sum_{j=0}^{m} \sum_{k=0}^{m} a_{j} a_{k} E\left\{g_{j} g_{k}\right\} \\
=a R a^{T}, a \neq 0, a \\
=\left(a_{0}, \ldots, a_{m}\right)
\end{aligned}
$$

It is seen that random variables $g_{j}(x), j=$ $0,1, \ldots, m$ are linearly independent according to assumption 1), the left-hand of (13) is equal to zero if and only if at $a=0$, consequently $R$ is positive defined matrix, therefore $\operatorname{det} R \neq 0$ and condition (10) is satisfied.
The satisfability of (9) and (10) indicates that the implicit function theorem [13] can be applied to solvability of (6) with respect to $\lambda_{0}, \lambda_{1}, \ldots, \lambda_{m}$.

The obtained results for solvability of (6) can be expressed in the following theorem.

Existence theorem. Let us the following conditions are satisfied:

(1) Moment functions $g_{j}(x), j=0,1,2, \ldots, m$ are linearly independent;

(2) The inequality $n>m$ is satisfied;

(3) Moment values $\tilde{\mu}_{j}, j=0,1, \ldots, m$ are obtained by virtue of given fuzzy values $\tilde{\mu}_{A}\left(x_{i}\right), i=$ $0,1, \ldots, n$ and $g_{j}(x), j=0,1, \ldots, m$ in the form of equalities

$$
\sum_{i=0}^{n} g_{j}\left(x_{i}\right) \tilde{\mu}_{A}\left(x_{i}\right)=\tilde{\mu}_{j}, j=0,1, \ldots, m .
$$

Then, Minimum Cross Fuzzy Entropy Problem $(\operatorname{Minx}(\mathrm{F})$ EntP) which consists of minimizing cross fuzzy entropy measure (1) with respect to membership functions $\mu_{A}(x)$ with finite number of the fuzzy values $\mu_{A}\left(x_{i}\right), i=0,1, \ldots, n$ and for a priori membership function $\mu_{B}(x)$ with finite number of the fuzzy values $\mu_{B}\left(x_{i}\right), i=0,1, \ldots, n$ subject to constraints (2) has a solution $\left(\mu_{A}\left(x_{0}\right), \mu_{A}\left(x_{1}\right), \ldots, \mu_{A}\left(x_{n}\right)\right)$.

\section{Evaluation of Lagrange multipliers for Minimum Cross Fuzzy Entropy Problem}

From the proof of Existence theorem, it is indicated that evaluation of Lagrange multipliers occupies very important place. For this reason, it is required to consider this problem in more detail. One of basic stages of application of numerical methods is the choice of any initial point.

The mean problem consists of solving system of equations (6) with respect to $\lambda_{0}, \lambda_{1}, \ldots, \lambda_{m}$ by starting any initial point $\left(\tilde{\lambda}_{0}, \tilde{\lambda}_{1}, \ldots, \tilde{\lambda}_{m}\right)$. Mentioned point is obtained by following way. From $\left(2_{1}\right)$ follows (7) and from $\left(2_{2}\right) \tilde{\mu}_{A}\left(x_{i}\right), i=0,1, \ldots, m$ are obtained in the form of $\left(2_{3}\right)$, later $\left(2_{3}\right)$ is taken into 
account in (8). Solving (8) with respect to $\lambda_{0}, \lambda_{1}, \ldots, \lambda_{m}$ and showing obtained values as $\tilde{\lambda}_{0}, \tilde{\lambda}_{1}, \ldots, \tilde{\lambda}_{m}$ it is seen that these values satisfy (9). Consequently to solve system (6) by some numerical method $\left(\tilde{\lambda}_{0}, \tilde{\lambda}_{1}, \ldots, \tilde{\lambda}_{m}\right)$ can be taken as initial point .

6. Minimum Cross Fuzzy Entropy Value

In Section 4, minimization of cross fuzzy entropy measure (1) is realized by Lagrange multipliers method and membership function $\mu_{A}(x)$ which gives minimum value to (1) is expressed by formula (4).

By virtue of formula (4) from (1), after taking elementary transformations follows that

$$
\begin{aligned}
& \min D\left(\mu_{A}: \mu_{B}\right) \\
& =-\sum_{j=0}^{m} \lambda_{j} \mu_{j} \\
& +\sum_{i=0}^{n} \ln \frac{e^{\sum_{j=0}^{m} \lambda_{j} g_{j}\left(x_{i}\right)}}{\mu_{B}\left(x_{i}\right)+\left(1-\mu_{B}\left(x_{i}\right)\right) e^{\sum_{j=0}^{m} \lambda_{j} g_{j}\left(x_{i}\right)}}
\end{aligned}
$$

The formula (14) represents minimum value of cross fuzzy entropy measure (1). It is seen that $\min D\left(\mu_{A}: \mu_{B}\right)$ depends on Lagrange multipliers $\lambda_{0}, \lambda_{1}, \ldots, \lambda_{m}$, moment functions $g_{0}(x), g_{1}(x), \ldots, g_{m}(x)$ and moment fuzzy values $\mu_{0}, \mu_{1}, \ldots, \mu_{m}$.

\section{Some Generalized Cross Fuzzy Entropy Optimization Problems}

Before it is showed that minimum value of cross fuzzy entropy optimization measure $D\left(\mu_{A}: \mu_{B}\right)$ is represented by formula (14) in the dependency of moment functions $g_{0}(x), g_{1}(x), \ldots, g_{m}(x)$, Lagrange multipliers $\lambda_{0}, \lambda_{1}, \ldots, \lambda_{m}$, and moment fuzzy values $\mu_{0}, \mu_{1}, \ldots, \mu_{m}$. Let us $g=\left(g_{0}, g_{1}, \ldots, g_{m}\right)$ be vector moment functions with components $g_{0}, g_{1}, \ldots, g_{m}$. If we take into account that according to condition (2) both Lagrange multipliers $\lambda_{0}, \lambda_{1}, \ldots, \lambda_{m}$ and moment fuzzy values $\mu_{0}, \mu_{1}, \ldots, \mu_{m}$ are generated by moment vector fuction $g$ and given fuzzy values of $\mu_{A}\left(x_{i}\right), i=0,1, \ldots, n$, then the $D\left(\mu_{A}: \mu_{B}\right)$ defined by formula (14) can be expressed as a functional $U(g)$ depended on moment vector fuction $g$. That is to say

$$
\begin{aligned}
& U(g)=\min D\left(\mu_{A}: \mu_{B}\right) \\
& =-\sum_{j=0}^{m} \lambda_{j} \mu_{j} \\
& +\sum_{i=0}^{n} \ln \frac{e^{\sum_{j=0}^{m} \lambda_{j} g_{j}\left(x_{i}\right)}}{\mu_{B}\left(x_{i}\right)+\left(1-\mu_{B}\left(x_{i}\right)\right) e^{\sum_{j=0}^{m} \lambda_{j} g_{j}\left(x_{i}\right)}}
\end{aligned}
$$

According to [14-18], let $K$ be the compact set of moment vector functions $g(x) . U(g)$ reaches its least and greatest values in this compact set, because of its continuity property. For this reason,

$$
\min _{g \in K} U(g)=U\left(g^{(1)}\right) ; \max _{g \in K} U(g)=U\left(g^{(2)}\right) .
$$

Consequently,

$$
U\left(g^{(1)}\right) \leq U\left(g^{(2)}\right)
$$

Distributions

$\mu^{(1)}=\left(\mu^{(1)}\left(x_{0}\right), \mu^{(1)}\left(x_{1}\right), \ldots, \mu^{(1)}\left(x_{n}\right)\right)$ and $\mu^{(2)}=\left(\mu^{(2)}\left(x_{0}\right), \mu^{(2)}\left(x_{1}\right), \ldots, \mu^{(2)}\left(x_{n}\right)\right) \quad$ corresponding to the moment functions $g^{(1)}(x)$ and $g^{(2)}(x)$ respectively, we call as $\operatorname{MinMinx}(\mathrm{F})$ Ent and $\operatorname{MaxMinx}(\mathrm{F})$ Ent distributions. Methods obtaining distributions MinMinx(F)Ent and MaxMinx(F)Ent we call as MinMinx(F)EntM and $\operatorname{MaxMinx}(F) E n t M$, respectively.

Now, MinMinx(F)EntM and MaxMinx(F)EntM for a finite set of characterizing moment functions can be defined in the following form.

Let $K_{0}=\left\{g_{1}, \ldots, g_{r}\right\}$ be the set of characterizing moment vector functions and all combinations of $r$ elements of $K_{0}$ taken $m$ elements at a time be $K_{0, m}$. We note that each element of $K_{0, m}$ is vector $g$ with $m$ components. Note that the number of vectors $g$ is equal to $\left(\begin{array}{c}r \\ m\end{array}\right)$.

Solving the $\operatorname{MinMinx}(F) E n t$ and $\operatorname{MaxMinx}(F) E n t$ problems require to find vector functions $\left(g_{0}, g^{(1)}(x)\right)$ and $\left(g_{0}, g^{(2)}(x)\right)$, where $g_{0}(x) \equiv$ $1, g^{(1)} \in K_{0, m}, g^{(2)} \in K_{0, m} \quad$ minimizing and 
maximizing functional $U(g)$ defined by (15). It should be noted that $U(g)$ reaches its minimum (maximum) value subject to constraints (2) generated by function $g_{0}(x)$ and all $m$-dimensional vector functions $g(x), g \in K_{0, m}$. In other words, minimum (maximum) value of $U(g)$ is least (greatest) value of values $U(g)$ corresponding to $g(x), g \in K_{0, m}$. In other words, MinMinx(F)Ent (MaxMinx(F)Ent) is distribution giving minimum (maximum) value to functional $U(g)$ along of all distributions generated by $\left(\begin{array}{c}r \\ m\end{array}\right)$ number of moment vector functions $g(x), g \in K_{0, m}$. Mentioned distributions can be denoted by (MinMinx(F)Ent $)_{\mathrm{m}}$ and $(\operatorname{MaxMinx}(\mathrm{F}) \mathrm{Ent})_{\mathrm{m}}$.

$$
\text { If }\left(g_{0}, g^{(1)}(x)\right)\left(\left(g_{0}, g^{(2)}(x)\right)\right) \text { gives the minimum }
$$
(maximum) value to $U(g)$ then distributions $\mu^{(1)}=\left(\mu^{(1)}\left(x_{0}\right), \mu^{(1)}\left(x_{1}\right), \ldots, \mu^{(1)}\left(x_{n}\right)\right) \quad$ and $\quad \mu^{(2)}=$ $\left(\mu^{(2)}\left(x_{0}\right), \mu^{(2)}\left(x_{1}\right), \ldots, \mu^{(2)}\left(x_{n}\right)\right) \quad$ corresponding to $\left(g_{0}, g^{(1)}(x)\right),\left(g_{0}, g^{(2)}(x)\right) \quad$ are called the MinMinx(F)Ent and MaxMinx(F)Ent distributions, respectively. MinMinx(F)Ent and $\operatorname{MaxMinx}(\mathrm{F})$ Ent methods represent fuzzy entropy distribution in the form of MinMinx(F)Ent and MaxMinx(F)Ent distributions. It should be noted that both distributions can be applied in solving proper problems in fuzzy data analysis.

\section{Conclusion}

In the present study the following results are achieved.

(1) It is proved the concavity property of cross fuzzy entropy measure and formulated a Minimum Cross Fuzzy Entropy Optimization Problem and proposed sufficient conditions for existence of its solution. Mentioned problem can be formulated as follows. In the set of membership functions satisfying the given moment constraints generated by given moment functions it is required to choose the membership function that is closest to a priori membership function in the sense of cross fuzzy entropy measure.

(2) A special functional $U(g)$ depended on moment vector functions $g$ is defined by applying Lagrange multipliers method.

(3) Minimum Cross Fuzzy Entropy Distribution (Minx(F)EntD) is distribution of fuzzy values $\left(\mu_{A}\left(x_{0}\right), \mu_{A}\left(x_{1}\right), \ldots, \mu_{A}\left(x_{n}\right)\right)$ minimizing cross fuzzy entropy measure subject to constraints generated by fixed moment vector function. Minimum Cross Fuzzy Entropy

Distribution $\left(\mu_{A}\left(x_{0}\right), \mu_{A}\left(x_{1}\right), \ldots, \mu_{A}\left(x_{n}\right)\right)$ can be considered geometrically as points $\left(x_{i}, \mu_{A}\left(x_{i}\right)\right), i=0,1, \ldots, n$ of membership function $\mu_{A}(x)$. Consequently, interpreting these points as experimental data it is possible to select formula, in other words membership function, in accordance on mentioned data by known methods.

Generalized Cross Fuzzy Entropy Optimization Methods in the form of $\operatorname{MinMinx}(F) E n t$ and $\operatorname{MaxMinx}(\mathrm{F})$ Ent methods are suggested on the basis of primary minimizing cross fuzzy entropy measure $D\left(\mu_{A}: \mu_{B}\right)$ for fixed moment vector function in order to obtain the special functional $U(g)$ with $\operatorname{Minx}(\mathrm{F})$ Ent values of cross fuzzy entropy measure and secondary optimization for mentioned functional with respect to moment vector functions. Distributions obtained by these methods are defined as $(\operatorname{MinMinx}(\mathrm{F}) \mathrm{Ent})_{\mathrm{m}} \quad$ and $\quad(\operatorname{MaxMinx}(\mathrm{F}) E n t)_{\mathrm{m}}$ distributions.

\section{References}

[1] L.A. Zadeh, Fuzzy sets, Information and Control 8 (1965) 338-353.

[2] L.A. Zadeh, Fuzzy sets and systems, in: Proceeding of the Symposium on Systems Theory, Polytechnic Institute of Brooklyn, New York, 1965, pp. 29-37.

[3] L.A. Zadeh, Probability measures of fuzzy events, Journal of Mathematical Analysis and Applications 23 (1968) 421-427.

[4] J.N. Kapur, H.K. Kesavan, Entropy Optimization Principles with Applications, Academic Press, New York, 1992. 
[5] J.N. Kapur, Measures of Fuzzy Information, Mathematiccal Sciences Trust Society, New Delhi,1997.

[6] C.E.Shannon, A mathematical theory of communications. Bell System Technical Journal 27 (1948) 379-623.

[7] A. De Luca and S. Termini, A definition of non-probabilistic entropy in setting of fuzzy set theory, Information and Control 20 (1971) 301-312

[8] D. Bhandari, and N.R. Pal, Some new information measures for fuzzy sets. Information Sciences 67 (1993) 209-228.

[9] J. Ye, Fault diagnosis of türbine base on fuzzy cross entropy of vague sets, Expert Systems with Applications 36 (2009) 8103-8106

[10] J. Ye, Fuzzy cross entropy of interval-valued intuitionistic fuzzy sets and its optimal decision-making method based on the weights of alternatives, Expert Systems with Applications 38 (2011) 6179-6183

[11] Xiang Li, Fuzzy cross-entropy, Journal of Uncertainty Analysis and Applications Sample doi:10.1186/s40467-015-0029-5.

[12] X. Chen, S. Kar, D.A. Ralescu,, Cross-entropy measure of uncertain variables, Information Sciences 201 (2012) 53-60.

[13] O. Parkash, P.K. Sharma and R. Mahajan, New measures of weighted fuzzy entropy and their applications for the study of maximum weighted fuzzy entropy principle, Information Sciences, 178,( 2008)2389-2395.

[14] R. Yager, On measures of fuzziness and negation, Part-I: membership in the unit interval, 1979.

[15] Vladimir A. Zorich, Mathematical Anaysis I, Moscow, Springer, 2002.

[16] S. Kullback, R.A. Leibler, On information nd suffiency, Annals of Mathematical Statitiscs, 4 (1951) 99-111

[17] A. Shamilov, Entropy, Information and Entropy Optimization, Turkey, 2009.

[18] A. Shamilov, A development of entropy optimization methods, WSEAS Trans. Math. 5 (2006) pp.-568-575.

[19] A. Shamilov, Generalized entropy optimization problems and the existence of their solutions, Phys. A: Stat. Mech. Appl. 382 (2007) 465-472.

[20] A. Shamilov, Generalized entropy optimization problems with finite moment function sets, Journal of Statistics and Management Systems, 13 (2010) 595-603.

[21] A. Shamilov, S. Ozdemir, N. Yilmaz, Generalized Entropy Optimization Methods for Survival Data, ALT2014: 5th International Conference on Accelerated Life Testing and Degradation Models, Pau, France, 2014, pp.174-183.

[22] Kwang H. Lee, First Course on Fuzzy Theory and applications, Springer, 2002. 